\title{
Dynamic changes in tRNA modifications and abundance during T- cell activation
}

Roni Rak ${ }^{1}$, Michal Polonsky ${ }^{2}$, Inbal Eizenberg ${ }^{2}$, Orna Dahan $^{1}$, Nir Friedman ${ }^{2 *}$, Yitzhak Pilpel ${ }^{1 *}$

${ }^{1}$ Department of Molecular Genetics,

${ }^{2}$ Department of Immunology,

The Weizmann Institute of Science, Rehovot, Israel.

*corresponding authors

Corresponding Authors:

Yitzhak Pilpel, Ph.D.

Weizmann Institute of Science

Hertzal st. Rehovot 76100, ISRAEL

Email: pilpel@weizmann.ac.il

Nir Friedman, Ph.D.

Weizmann Institute of Science

Hertzal st. Rehovot 76100, ISRAEL

Email: nir.friedman@weizmann.ac.il

Classification:

BIOLOGICAL SCIENCES; cell biology

\section{This PDF file includes:}

- Main Text

- Figures 1 to 5

- Supporting information: 4 supplementary Figures and 1supplementary tables 


\section{Abstract}

The tRNA pool determines the efficiency, throughput, and accuracy of translation. Previous studies have identified dynamic changes in the tRNA supply and mRNA demand during cancerous proliferation. Yet, dynamic changes may occur also during physiologically normal proliferation, and these are less characterized. We examined the tRNA and mRNA pools of Tcells during their vigorous proliferation and differentiation upon triggering of the $T$ cell antigen receptor. We observe a global signature of switch in demand for codon at the early proliferation phase of the response, accompanied by corresponding changes in tRNA expression levels. In the later phase, upon differentiation of the T cells, the response of the tRNA pool is relaxed back to basal level, potentially restraining excessive proliferation. Sequencing of tRNAs allowed us to also evaluate their diverse base-modifications. We found that two types of tRNA modifications, Wybutosine and $\mathrm{ms}^{2} \mathrm{t} 6 \mathrm{~A}$, are reduced dramatically during T-cell activation. These modifications occur in the anti-codon loops of two tRNAs that decode "slippery codons", that are prone to ribosomal frameshifting. Attenuation of these frameshift-protective modifications is expected to increase proteome-wide frameshifting during T-cell proliferation. Indeed, human cell lines deleted of a Wybutosine writer showed increased ribosomal frameshifting, as detected with a reporter that consists of a critical frameshifting site taken from the HIV gag-pol slippery codon motif. These results may explain HIV's specificity to proliferating T-Cells since it requires ribosomal frameshift exactly on this codon for infection. The changes in tRNA expression and modifications uncover a new layer of translation regulation during T-cell proliferation and exposes a potential trade-off between cellular growth and translation fidelity.

Keywords:

Transfer RNA, T-cells activation, tRNA-modifications, HIV

\section{Significance statement}

The tRNA pool decodes genetic information during translation. As such, it is subject to intricate physiological regulation in all species, across different physiological conditions. Here we show for the first time a program that governs the tRNA pool and its interaction with the transcriptome upon a physiological cellular proliferation- T-cells activation. We found that upon antigenic activation of T-cells, their tRNA and mRNA pools undergo coordinated and complementary changes, which relex when cells reduces their proliferation rate. We also found a reduction in two particular tRNA modifications that have a role in governing translation fidelity and frameshift prevention. This exposes a vulnerability in activated T-cells that may be utilized by HIV for its replication.

\section{Introduction}

Few cells in the adult mammalian body can proliferate under normal conditions. One example of a fundamental programmed proliferation processes is evoked in clonal expansion of selected cells of the adaptive immune system, following encounter with foreign antigens. Upon recognition of a cognate antigen, naive T-cells (and B-cells) undergo massive proliferation and differentiation, hence changing their status from arrested naive cells to highly proliferating effector cells ${ }^{1}$. The integrity of the immune response is dependent on the precise regulation of proliferation rates, number of cell-cycles and cell death. In addition, a portion of the population is differentiated into memory cells, which remain in the body for extended periods of time. 
A crucial part of cell proliferation is the ability to massively translate new proteins. Translation control was shown to regulate gene expression in the immune system ${ }^{2}$. However, the regulation of translation elongation, and especially tRNA availability was not explored. We have previously shown that genes that are upregulated in proliferating cancerous cells have a distinct translation program from arrested cells ${ }^{3}$. In particular, mRNAs corresponding to cell-autonomous functions, related to proliferating cells, are enriched with a specific set of codons, while mRNA of multicellular functions, related to non-dividing cells, are enriched with a different set of preferred codons. The tRNA pool in these cells was dynamically regulated and its dynamics corresponded to the changes in codon usage ${ }^{4}$. Following these observations, we asked how does the tRNA pool change in a natural proliferation process, and what are the contributions of the tRNA pool regulation to the integrity dynamics and of the immune response.

Beyond changes in expression level of tRNAs, these RNAs are among the most highly posttranscriptionally modified molecules in the mammalian transcriptome ${ }^{5,6}$ Essentially all tRNA molecules undergo diverse chemical modifications on many of their bases, and the various nucleotide modifications can affect tRNA folding and stability, amino acid loading, and codonanticodon base pairing of tRNAs ${ }^{7-11}$. Modifications around the anticodon loop of the tRNA are known to affect translation fidelity, speed, and tendency to frameshift ${ }^{11-14}$. Modification levels are regulated and can change in a dynamic manner across physiological conditions ${ }^{15-17}$, in cancer ${ }^{18,19}$, and during development ${ }^{20}$. Yet the dynamics, regulation and biological function, both at the molecular and physiological level, of most such modifications are not well-characterized.

Here we set to investigate the control of the tRNA pool in a physiologically normal and programmed proliferation process, by following T-cells upon their antigen receptor activation. We examined the codon usage changes during T-cell activation and the corresponding changes in tRNA abundance. We also examined in parallel the post-transcriptional chemical base modifications of the tRNAs. We found that upon activation, T-cells reprogram their tRNA pool so-as to serve the altered codon usage demand of the proliferation related genes. Interestingly though, at the later stage of the response, when T-cells differentiate, the tRNA response is relaxed back towards base level, where it is no longer adapted to the proliferation related codon usage. Further, we found that at the pick of their proliferation, T-cells exhibit a sharp decline of two tRNA base modifications. Both of these modifications are known to protect the ribosome from -1 frameshifting at "slippery" frameshift-prone codons. Interestingly, the reproduction of human immunodeficiency virus-1 (HIV-1), which preferentially targets proliferating T-cells, depends exactly on such -1 frameshifting that occurs on one of these slippery codons ${ }^{21}$. We indeed found that knockout of the tRNA modification enzyme of one of the modifications, in human cells, induced a frameshift at the HIV frameshifting slippery codon sequence. While the biological reason for reduction of this modification in T-cells is still unknown, we hypothesize that it may represent a trade-off between translation fidelity and speed, a requirement for fast proliferation 


\section{Results}

\section{Effective Codon usage changes from differentiation signature to proliferation signature} and back during T-cell activation

An important aspect of translation regulation is the dynamic change of supply (tRNA and anticodons) and demand (mRNA and codons). To study translation regulatory dynamics during a central process of mammalian cellular proliferation and differentiation we followed T-cells as they are triggered to switch from a naïve to an activated state. Naïve CD4+ T-cells were isolated from mice spleen and were activated using anti-CD3/anti-CD28 activation beads (see M\&M). Samples were taken at the naïve stage ("time point zero"), and after $20 \mathrm{~h}, 48 \mathrm{~h}$, and $72 \mathrm{~h}$. At the later time points (i.e. 48 and $72 \mathrm{~h}$ ) cells were sorted according to expression of the CD62L and CD44 markers which separate effector lymphocytes (CD62L-CD44+, hereby Eff) from precursor memory cells (CD62L+CD44+, hereby Mem). We sequenced both mRNA and tRNA from each sample using two distinct protocols (Fig $1 \mathrm{~A}$, see M\&M).

We first characterize changes in mRNA codon usage by examination of the change in representation of each of the 64 codon types in the mRNA pool in each time point. The "demand" for tRNA by each codon type in the transcriptome is computed as the sum, over all mRNAs, of the product of mRNA expression level and the number of appearances of each codon in each gene ${ }^{3}$. We found that relative to the naïve cells, activated proliferating cells show an increase in representation of the codons defined before by Gingold et al. ${ }^{3}$ as the "proliferation codons" signature (Fig. 1B). The "proliferation codons" tend to mostly end with A or T at the third codon position, in contrast to the "differentiation codons" which tend to have $\mathrm{G}$ or $\mathrm{C}$ at their $3^{\text {rd }}$ positions. Although the enrichment in "proliferation codons" is seen in all samples, when compared to naïve cells, the difference is maximimal at the $20 \mathrm{~h}$ sample, and it is then reduced when cells differentiate into memory precursors (72h-high CD62L, Mem), Fig. 1B.

We characterized each time point sample based on the average codon usage of the genes expressed in it, normalized by their expression level. Fig. 1C shows a 2D projection based on Principal Component Analysis (PCA) of the codon usage by samples matrix. In this projection, closely projected time points have transcriptomes with similar codon usage. The analysis shows a marked change in codon usage at $20 \mathrm{~h}$, relative to the naïve population, and a gradual return towards base levels when cells differentiate into memory cells at 72 hours. Which genes derive the codon usage trend? We first checked the genes that belong to the Gene Ontology categories associated with the different stages of the cell cycle (G0-early G1, G1, S, G2M, M). We found that the first PC of the codon usage PCA is in high correlation with the expression of S-phase genes $(R=0.95)$. The correlation to $G 1, G 2 / M$, and $M$-phase $G O$ categories was also high, but lower then S-phase ( $R=0.67, R=0.74, R=0.55$, respectively. (Fig. 1D,E and Fig. S1)). Apart from cell-cycle related GO categories, the most correlative GO categories are: "negative regulation of oxidative stress-induced cell death", "regulation of DNA metabolic process", "cellular respiration" and "purine ribonucleoside monophosphate metabolic process"(see sup. Table 1). We conclude that induction of genes belonging to these functional categories drives the proliferation-codon usage signature that we observe in activated T-cells. 
Induction of a proliferation tRNA signature is followed by relaxation at the differentiation phase

We next set to examine what are the changes in the tRNA pool and if they correspond to the changes in demand as reflected by the codons usage, adapting a recently developed tRNA sequencing protocol ${ }^{22}$ (See Materials and Methods for further details). In the downstream analysis we grouped together read counts of all tRNAs of the same anticodon that have at least a minimal tRNAScan prediction score (tRNAscan- $\mathrm{SE}^{23}$ ) above 50 . We calculated the expression of each such isoacceptor group as the sum of read counts mapped to each of its corresponding genes. We found that 20 hours after activation of the cells, the tRNAs that decode the A/T ending codons are upregulated compared to naïve T-cells (Fig. $2 A$, ranksum $p$-val<0.05). We did not observe significant difference in expression fold change between tRNAs that code for $A / T$ ending codons and $\mathrm{G} / \mathrm{C}$ ending codons in any of the other time-points.

In parallel, we examined codon usage programs of various functional gene sets. For that we projected all mouse genes' GO categories on a PCA plane, based on the average codon usage of all the genes that belong to each category (Fig. 2B,C). The location (but not the coloring) of the $\mathrm{GO}$ categories is identical in all four sub-figures, as it reflects codon usage only. In similarity to the human genes analysis ${ }^{3}$, on the right hand side are proliferation-related functionality such as the cell cycle genes, and functions related to gene expression such as the ribosome, DNA replication machinery genes, while on the left side are differentiation related functions(figure S2). This analysis clearly shows the marked difference in codon usage of genes that fulfil proliferation and differentiation functions. To examine correspondence between changes in supply and demand we colored the GO categories in each time point once based on change in mRNA expression (upper panel) and once based on change in tRNA supply (lower panel). Shown here are time points 20 and 72 hours (Fig. $2 \mathrm{~B}$ and $2 \mathrm{C}$ respectively). Examining the changes in mRNA expression level between 20 and 72 hours to time point zero reveals that the proliferation-related GO categories on the right hand side of the PCA projection are strongly induced at the mRNA level. The signature is stronger at 20 hours, reflecting reduction in proliferation at 72 hours. We next analyzed dynamic changes in tRNA supply of the genes belonging to each $\mathrm{GO}$ category through analysis of the adaptation of their codon usage to the changing tRNA pool. We used the tAI score ${ }^{24}$ (see Materials and Methods) which calculates codon adaptiveness based on the tRNA availability. Here, we determine the tRNA availability based on the read count for each anticodon. This allows us to determine translation efficiency for each codon type in a dynamic fashion, across the process of T-cell activation. We then calculated the translation efficiency for each gene, as a (genometric) average of all its codons, and we then averaged this gene score across all the genes in each GO category. We next compute the ratio of this tAl score for each GO category given the tRNA pool at a given time point, normalized to the score computed based on the time zero tRNA pool. The ratio is higher than 1 at a given time point during T-cell activation for GO categories whose genes have a codon bias that correspond to tRNAs that are induced in that time point. 
We found that in the activated cells, at 20 hours after activation, the proliferation genes are induced at the mRNA level, and the tRNAs that correspond to their codon usage are induced too. Across the set of all GO categories there is a positive correlation between the change in $t A I$ value and change in mRNA expression (Fig. 2B lower panel). At 72 hours after activation memory cells (Fig. 2C) there is no such correlation. The proliferation-related genes are still induced at the mRNA level, but to a lesser extent compared to the 20 hours, and the tRNA pool's supply is no longer biased towards the codon usage of the genes that belong to these functionalities. In that respect the relationships between the tRNA and mRNA pool at 20 hours, but not in 72 hours of the T-cell activation process resemble the situation seen in diverse cancer types ${ }^{3}$. It is possible to speculate that such lack of correlation between supply and demand at the 72 hours time point might ensure that the pro-proliferation mRNAs that may still exist in the cell at this stage will not be translated efficiently, restraining potential undesired excessive proliferation.

\section{tRNA modifications involved in translation frame maintenance are reduced in activated T} -cells.

tRNA molecules are extremely rich with post-transcriptional RNA modifications, of which some can govern the stability, translation efficiency, decoding rate and fidelity ${ }^{7,10,25,26}$. Modifications can be detected using tRNA-seq as they can often cause failure in the reverse transcription reaction ${ }^{9}$. In modified positions, the reverse transcriptase can either abort the reaction, resulting in truncated fragments or it may introduce typical nucleotide mismatches compared to the genomic sequence. The fraction of RNA copies that carry the modification can thus be calculated as the sum of mismatches and RT-abortion truncated reads at the position, normalized to the total number of reads that map, either perfectly or not, to the gene's sequence. Modifications that do not interfere with the RT process are not detectable through this method. We note that the data that emerge from sequencing do not allow to detect the actual chemical nature of the modified nucleotide, only relative changes in the level of editing, but crossing the read data with databases of annotated modifications may allow to infer the nature of modification and to detect changes in the fraction of edited transcripts.

Here we compared all suspected sites of modification observed in our tRNA sequencing data during T-cell activation, against the MODOMICS database ${ }^{5}$. For mouse, twelve tRNA species are annotated with their modifications in this database, and in total they represent 18 modification types, in 196 modification positions in total. Through changes in tRNA reads we have been able to detect 5 out of 18 annotated modifications ( $\mathrm{m} 1 \mathrm{~A}, \mathrm{~m} 1 \mathrm{G}, \mathrm{m} 2,2 \mathrm{G}, \mathrm{l}$, and wybutosine), in 24 known positions. In addition, 615 un-annotated sites appear to be at least minimally modified (as detected either by abortion of RT or by apparent nucleotide sequence change, in at least $10 \%$ of the reads). Further, we could quantify changes in modification levels throughout the Tcell activation process by comparing modification level between naïve and activated T-cells. We examine separately the modifications around the anticodon (and one nucleotide upstream and downstream), which has a higher effect on tRNA decoding role, and the modifications upstream 
and downstream to the anticodon (Fig. 3A and Fig. S6), which are involved in many other aspects of tRNA biology ${ }^{27}$.

We found that the level of most modifications remained constant during the T-cell activation process (Fig. 3A). However, two tRNAs (LysTTT and PheGAA) show marked reduction in modification level in activated cells at 20 hours compared to the naïve cells, on position 37 (1nt upstream from the anticodon) (Fig. $3 \mathrm{~B}$ left panel). For comparison, the downstream modification m1A 58 remained constant for both tRNAs (Fig. $3 \mathrm{~B}$ right panel). The preservation of the other modifications indicates that these tRNAs maturate and are processed properly. We also detected changes in modification levels of ArgUCU at a position more distant from the anticodon, assumed to be $\mathrm{m}^{3} \mathrm{C} 32$ (figure S6A) .

Interestingly, both of the observed anticodon modifications are involved in decoding of "slippery codons" which are known to be involved in ribosomal frameshift. Position 37 PheGAA is annotated in MODOMICS to have the wybutosine modification. Wybutosine stabilizes codonanticodon interactions, and prevents -1 frameshifts caused by polyuridine (phe-GAA decodes both UUC and UUU codons) ${ }^{13,28}$. Position 37 at LysTTT tRNA is not annotated for mouse in MODOMICS. Our data support the presence of the bulky modification 2-methylthio-6threonylcarbamoyl-A $\left(\mathrm{ms}^{2} \mathrm{t}^{6} \mathrm{~A}\right)$ as shown before in mouse ${ }^{14}$ and human ${ }^{5,29}$. This modification was shown to cause RT abortion in human ${ }^{9}$. Lysine codon stretches, particularly AAA, are known to be involved in - $1 \mathrm{frameshift}^{30}$. In bacteria, tRNA ${ }^{\text {Lys }}$ is responsible for frameshifting at dnaX gene, which occurs in $50 \%$ of the translation events ${ }^{31}$.

Due to limited information on the modification on LysTTT, we will focus here on the modification on PheGAA. We first wished to substantiate the hypothesis that this tRNA indeed carries a wybutosine modification. For that, we used CRISPR-cas9 to knock out the modification enzyme, the gene tyw1. This enzyme catalyzes the first reaction in the chemical chain that converts the $1 \mathrm{G}$ modification on position 37 of PheGAA to wybutosine. We generated clones of HeLa tyw1 knock-out-cells using two alternative gRNAs that target the second or third exon of tyw1 gene. Indeed the wybutosine modification level was reduced on PheGAA by 20 to $30 \%$, from an initial level higher than $>90 \%$ (Fig. 4C). Interestingly, the total levels of the PheGAA tRNA is elevated upon this knock-out (Fig. 4D), suggesting a feed-back loop that could sense the reduction in the modified tRNA level and respond by elevating transcription of the tRNA molecules. Alternatively, it is possible that the modification de-stabilizes the tRNA.

\section{Reduced Wybutosine modification level increases ribosomal frameshift level}

To test the influence of the wybutosine modification on frameshift level we used a fluorescent protein-based frameshift reporter. The reporter is constructed from two fluorescent proteins, the first is translated from the "zero" frame and the second from the "-1" frame. The nucleotide sequence in the linker between the two fluorescent genes is assayed for its effect on ribosomal frameshifting level. Since the down-stream protein can only be made if the linker sequence induces frameshifting, intensity of that protein's fluorescence is a measure of frameshift level. The intensity of the up-stream protein is independent of frameshift and it is used for normalization. We have recently created a library of such dual fluorescent proteins connected with diverse candidate linkers ${ }^{32}$. To select from this library one biologically meaningful linker sequence we returned to the biology of activated T-Cells. We recall that infectivity of HIV-1 is 
primarily focused on activated T-Cells, and is heavily dependent on the UUU (-1) frameshift for translation of its polymerase enzyme ${ }^{21,33}$. We have thus chosen the HIV gag-pol frameshift sequence as the linker for our reporter (Fig. 4A).

We transfected the tyw1 KO cells with the fluorescent frameshift reporter and measured the frameshift level. We found that the frameshift level is significantly elevated by $6 \%$ in the mutant compared to the wild-type. This indicates that down-regulation of wybutosine modification can indeed exert an effect on frameshift in general and that it might affect production of HIV polymerase, which depends on ribosomal frameshift.

\section{A Trade-off between translation fidelity and speed}

Why do activated T-cells reduce the level of the two tRNA modifications that have a role in reducing ribosomal frameshift? We hypothesize that the T-cell system may have evolved to trade-off translation fidelity and speed, as discussed in other contexts ${ }^{34,35}$. One possibility is that this reduction in modification allows cells to translate proteins more rapidly, a necessity under conditions that require rapid growth in biomass production, towards cellular proliferation. Under such conditions, we reason, the system evolved to compromise translation fidelity, i.e. to feature enhanced frameshift. This hypothesis raises the prediction that under such a trade-off scenario, genes involved in T-cell activation would avoid the slippery codons (UUU) in favor of synonymous codons (UUC) that are not prone to frameshifting, thus reducing translation error rate. We examined each mouse gene for its tendency to prefer either UUU or UUC for encoding Phe, and ranked all genes according to this codon bias. Strikingly, and in line with our predictions, we found that the genes with the highest UUC/UUU ratio have several functionalities involved in T-cell and other immune functions (Fig. 5A). As a negative control, we examined the other "2-box" codon duplets in the genetic code, i.e. all pairs of synonymous codons of the type XXU XXC. For each such pair, we computed across all genes, the XXU/XXC preference score and ranked the genes according to the extent of XXU avoidance. None of the other eight codon pairs showed an enrichment for any functionality related to T-Cells (Table s1). Avoidance of frameshift-prone codons in the genes expressed in proliferating T-cells may reduce undesired frameshifting events that can occur due to reduction in the tRNA modification.

\section{Disscussion}

In this work we studied the tRNA pool and its interaction with the MRNA pool in a natural physiological context of massive cellular proliferation, that of activated T-cells. The interplay between the tRNA and mRNA pool in cells was shown to govern proteome-wide translation

$4,36,37$. Previous studies have shown that the mammalian proliferation state affects the cellular tRNA pool, but most studies were focused on cancerous proliferation ${ }^{11,38}$. Non-cancerous, physiologically normal proliferation may obey different dynamics. Normal cellular proliferation must be a restrained process, one that actually must have evolved to avoid undesired cancerous 
transformation. Normal proliferation evolved over long organismal evolutionary time scales, as opposed to cancer, which operates selection over short time scales of cellular somatic evolution. The motivation to inspect the tRNA pool under conditions of normal proliferation is thus clear.

Indeed, we find that early on upon stimulation, proliferating T-cells feature transcription activation of the proliferation translation program. Induced mRNA coding genes that fulfil the proliferation needs of cells represent a proliferation codon usage program and they are rather adequately served by the proliferation-oriented tRNAs that are largely induced at this stage. Yet, after the pick of proliferation ( $72 \mathrm{hr}$ in this experiment), while many of the proliferating mRNA transcripts are still present, the tRNA pool appears to have relaxed back and it might no longer translate as efficiently the pro-proliferation transcripts. At this time-point the difference between the effector T-cells and the memory T-cells is interesting, while the memory cells' tRNA pool appears almost fully relaxed, the effectors still show some residual induction of the proliferation tRNA pool signature. This is in line with reduced proliferation rate that was observed for memory precursors compared with effector T cells during influenza infection ${ }^{39}$. Thus, the faster decline in expansion of memory $T$ cells might be regulated in part by dynamics of tRNA expression that result in imbalance between codon supply and demand.

This study goes beyond changes in tRNA levels, as it inspects dynamics in tRNA modifications during the T-cell activation process. While most modifications on most tRNAs do not change, we found two modification that are one nucleotide downstream of the anti-codon loops of two tRNAs, which drop significantly during the pick of proliferation, and are relaxed back towards base level when cells differentiate into memory cells. Interestingly, these two tRNAs decode "slippery codons" that are otherwise prone for ribosomal frameshifting. It thus appears that during the pick of proliferation, cells trade off translation adequacy, and maybe even run into the risk of ribosomal frameshifting.

Why do activated T-cells reduce the level of the two tRNA modifications that have a role in reducing ribosomal frameshift? One possibility is a putative beneficial effect of frameshifting on these cells at the proliferation stage. It is possible to speculate that ribosomal frameshifting might enhance phenotypic variability of immunological repertoires, such as the T-cell receptor. A more conservative alternative is that the T-cell system may have evolved to balance the tradeoff between translation fidelity and speed, as has been discussed in other cellular contexts ${ }^{34,35}$. According to this possibility, reduction in modification allows cells to translate proteins more rapidly, i.e. the presence of the modifications stabilize the codon-anticodon pairing and increases fidelity ${ }^{14,28,40,41}$ This is a necessity under conditions that require rapid growth in biomass, towards extensive cellular proliferation. In support of this possibility, we indeed found that genes involved in immune response tend to avoid the use of the slippery codon UUU. Notwithstanding, despite the apparent avoidance of slippery codons in the immunity-related genes, other genes expressed in T-cells might still be subject to elevated frameshifting upon reduction in the modification. Thus, the second prediction of the speed-fidelity tradeoff model is that if indeed T-cells experience a general, perhaps non-specific, increase in frameshifting, they could suffer from a proteotoxic stress. Interestingly we found that the proteasome, both the catalytic and regulatory sub-units, is induced around $20 \mathrm{~h}$ upon activation, followed by relaxation at later time points, suggesting that a proteotoxic stress is experienced by the proliferating cells (Fig 1S, bottom panel) ${ }^{42,43}$. The model that thus emerges is that T-cells reduce the level of two 
tRNA modifications that when present prevent frameshifting at slippery codons. In doing so, they might trade-off translation fidelity and speed. They protect their most crucial and cell specific genes from containing the slippery codons, but they may suffer from otherwise nongene specific frameshifting activity.

Most observations in this study were done in mouse T-cells. Yet, assuming the T-cell proliferation in human cells features similar reduction of the same tRNA modifications, would lead to the hypothesis that the increase in the potential for ribosomal frameshifting may also occur in proliferating human T-cells. Interestingly, activated human T-cells are the infection targets of HIV, which necessitates calibrated frameshifting rates on the UUU slippery codon between its gag and pol ORFs ${ }^{21}$. Thus, while T-cells must be reducing tRNA modifications for their own needs and interest, it is tempting to speculate that HIV takes advantage of this dynamics that is utilized by the virus as a vulnerability.

\section{Material and methods}

\section{T-Cell isolation and FACS sorting}

Naïve cells were extracted from six spleens of B6 mice using the StemCell CD62L+CD44+ kit (female, 7 weeks, 6-9\% naive). Each two spleens were pooled to a total of 3 samples extracted separately. A portion from the naïve state was taken to FASC sorted for live cells followed by RNA extraction. The remaining naive cells were activated by addition of anti-CD3/anti-CD28 activation bead (1:1 bead:cell ratio, thermoFisher Scientific Cat\#11131D)

Cells were grown at $0.25 \mathrm{cell} / \mathrm{ml}$ in 24 wells, and were collected at $20 \mathrm{~h}, 48 \mathrm{~h}$, and $72 \mathrm{~h}$ after activation. At $20 \mathrm{~h}$ only live cells were sorted. At 48 and $72 \mathrm{~h}$ cells were sorted into CD62L+CD44+ (memory T-cells) and CD62L-CD44+(effector T-cells). The cells were stained as followed, and analyzed using ARIAIII FACS:

\begin{tabular}{|l|l|}
\hline Antibody & fluorophore \\
\hline CD4 & Pacific-Blue \\
\hline CD62L & PE/Cy7 \\
\hline CD44 & FITC \\
\hline CD69 & PE \\
\hline CD25 & APC \\
\hline
\end{tabular}

\section{RNA extraction}

Sorted cell and unsorted culture cells were washed with PBS twice. The supernatant was removed and cells were re-suspended in TRIzol (thermofisher scientific). RNA was extracted according to manufacture protocol. 


\section{Cell culture}

HeLa cells (ATCC; CRL-3216) were grown in DMEM high glucose (Biological Industries; 01-052-

1A) supplemented with $10 \% \mathrm{FBS}, 1 \%$ penicillin/ streptomycin (P/S) and $1 \% \mathrm{~L}$-Glutamine.

Transfections were done using JetPEI transfection reagent (polyplus transfection) using standard manufacture protocol.

\section{Generation of TYW1 knockout}

HeLa cells were seeded onto $10 \mathrm{~cm}$ plates and growing for $24 \mathrm{~h}$ until reaching a confluence of approximately $70 \%$ (seeding $\sim 2 * 10^{6}$ cells). $5 \mu \mathrm{g}$ of LentiCRISPR V2 vector (addgene \#52961) containing either one of two sgRNA for TYW1 gene or non-targeting control sgRNA was transfected to cells using JetPei . Guide RNA sequences was chosen from Predesigned Alt-R ${ }^{\circledR}$ (IDT), guide sequences are:

\section{TWT1 guide2: 5'-AACCACTCTGCACTTTCAGT,-3'}

TWT1 guide 3: 5'-TCTTCACTGGAGCTCTCAAA-3'.

Non-targeting guide: 5'- TTTCGTGCCGATGTAACAC-3'.

Four hours after transfection, the medium was replaced with a medium. Two days after the transfection, DMEM medium containing $2 \mu \mathrm{g} / \mathrm{ml}$ puromycine was added to the transfected cells, refreshed every day for 3 days, until non-transfected cells reach $100 \%$ death. Transfected cells were diluted in 96 wells plate to a concentration of a single cell per well and grown, grown for two weeks to generate single clones. Knock-out of TYW1 gene in each clone was validated using sanger sequencing with the following primers:

For TYW1- guide 2 validation:

Fwr: 5'-GGTGCCCTTTGTTCTTTGAGATG-3'

Rev: 5'-GTGTGCGTGTGTGCACGTG-3'

For TYW1- guide 3 validation:

Fwr: 5'- GAGAGCTTTGCCTTTGCCCC -3'

Rev: 5'- CTTTTCTTTCTTCACATGATCC -3'

We arbitrary selected clones that show editing in the PAM position for downstream analysis.

\section{Frameshift assay}

Control and TYW-1 knock-out HeLa cells were seeded onto 6-well plates such thaT-cell confluence will be approximately $70 \%$ the next day. ( 150,000 cells). Cells were transiently transfected with frameshift reporter plasmids. The plasmids expresses mCherry, followed by HIV gag-pol frameshift signal, followed by GFP out of frame (-1). As a control we used in-frame GFP 
and out of frame $(+1)$ GFP in the same plasmid context. The plasmids were generous gift from Martin Mikl ${ }^{32}$. The cells were analyzed using ATTUNE FACS 48h after transfection. Frameshift rate was calculated as GFP/mCherry ratio.

\section{mRNA sequencing}

mRNA sequencing was preform using MARS-Seq protocol as described ${ }^{44}$. Libraries were sequenced on illumina NEXTseq platform.

Reads were trimmed using cutadapt (DOI: 10.14806/ej.17.1.200) and mapped to genome /shareDB/iGenomes/Mus_musculus/UCSC/mm10/Sequence/STAR_index using STAR (DOI: 10.1093/bioinformatics/bts635) v2.4.2a (default parameters).

The pipeline quantifies the genes annotated in RefSeq (that have expanded with 1000 bases toward 5' edge and 100 bases toward 3' bases):

/home/labs/bioservices/services/ngs/support/gtf/mm10.genes.3utr.gtf.

Counting was done using htseq-count (DOI: 10.1093/bioinformatics/btu638) (union mode).

Further analysis is done for genes having minimum 5 read in at least one sample.

Normalization of the counts and differential expression analysis was performed using DESeq2 (DOI: 10.1186/s13059-014-0550-8) with the parameters: betaPrior=True, cooksCutoff=FALSE, independentFiltering=FALSE. Raw $P$ values were adjusted for multiple testing using the procedure of Benjamini and Hochberg.

Pipeline was constructed using Snakemake (DOI: 10.1093/bioinformatics/bts480).

\section{tRNA sequencing and read analysis}

tRNA sequencing protocol was adapted from Zheng et al., 2015 (Zheng et al., 2015) with minor modification. The protocol involves the use of a highly persistent and thermo-stable reverse transcriptase. The primers are DNA-RNA hybrids that have overhang of $T$, which enable reverse transcription of A ending RNAs. This leads to enrichment of mature tRNAs, due to their shared CCA tail. We isolated small RNAs of ( $<200 \mathrm{bp}$ ) from the total RNA isolated using SPRI-beads (Agencourt AMPure XP, Beckman Coulter; A63881), using dual side size-selection protocol. First RNA and beads were mixed at 1:1.8 ratio, collection the supernatant. The small RNA was isolated by mixing the clear supernatant with bead at 1:0.8 ratio, with addition of X1.34 isopropanol. Reverse transcription was done using TGIRT TM -III Enzyme (InGex, LLC), with the indicated primers. $3^{\prime}$ adaptor was ligated to the cDNA using T4 ligase (NEB; M0202S). The cDNA was purified using Dynabeads myOne SILANE (life Technologies; 37002D) after each step. The library was amplified using NEBNext PCR mix and cleaned using SPRI-beads. Samples were pooled and sequenced using a 75bp single read output run on MiniSeq high output reagent kit.

Primer Reverse-transcription DNA 5'-CACGACGCTCTTCCGATCTT -3' . Reverse-transcription RNA 5'-rArGrArUrCrGrGrArArGrArGrCrGrUrCrGrUrG-3'. 3'-ligation adaptor 5'-

AGATCGGAAGAGCACA-3'

Read were trimmed using homerTool. Alignment to the genome and mature tRNAs gene sequence was done using Bowtie2 with parameters --very-sensitive-local. Reads aligned with 
equal alignment score to the genome and mature tRNAs were annotated as mature tRNAs. Reads aligned to multiple tRNA genes were randomly assigned when mapping to identical anticodons, and discarded from the analysis if aligned to different anticodons. Read count was done using BedTools-coverage count. Variant calling for detection of mutation and indels was done using samtools command "mpileup" with the parameters: "-A -q1 -d100000".

Using this tRNA sequencing protocol, 45\%-60\% (depending on the sample) out of the reads are mapped to mature tRNAs, the cytosolic or mitochondrial. Additional $1 \%-6 \%$ of the reads were aligned to pre-mature tRNAs. Out of 273 unique mature tRNA sequences in the mouse genome, 120-156 tRNA genes get sufficiently high read counts that allow their quantification. On the other hand, 116-141 tRNAs did not get sufficient number of reads (under 20 reads). All the tRNA genes with a low score tRNAScan score (that is, the tRNA prediction algorithm ${ }^{23}$ ), have low read counts, and can be considered as pseudo-tRNA (Fig. S4). The low read count indicates that those RNAs are either not expressed or cannot pass through the maturation process. Individual tRNAs were next grouped by anticodon. We calculated the gene copy number for each anticodon based on the number of tRNA genes in the genome, with prediction score above 50 (Fig. S2B). We found that although they exist in the genome, tRNAs of certain anti-codons, with low gene copy number, have very low expression. For all of those anticodons, the matching codons can be translated by wobble interactions with a different tRNA group. These results are similar to measurements done in human HEK293 cells and in the brain ${ }^{45}$, and they are in agreement with the predication that approximately a third of the tRNA genes in mouse are inactive in all tissues, and around $30 \%$ are tissue specific ${ }^{46}$.

\section{Codon usage calculation}

Codon usage and codon bias were calculated based on mRNA expression. We summed the usage of each codon in each gene multiplied by the expression of the gene.

$$
\{\text { CodonUsage }\}=\sum_{i=1}^{n} \text { codonFrequncy in gene } i \times m R N A \text { expressionofgene } i
$$

\section{Estimating Translational Efficiency in Terms of tRNA expression}

We generated a new measure of translational efficiency (implemented in Figure 2). Our measure is calculated similarly to the tAI measure of translation efficiency ${ }^{24}$, with one major change- we determine tRNA availability based on the read aligned to the indicated tRNA, as done in our previous work ${ }^{3}$. As such, the new measure can be computed for every condition. Since there are biases in the tRNA sequencing methods we employed this method as a comparative approach- we report fold-changes between conditions and not the actual value.

\section{Data sources}

Coding Sequences. The coding sequences of M. musculus were downloaded from the Consensus CDS (CCDS) project (ftp://ftp.ncbi.n/m.nih.gov/pub/CCDS/).

Classification of Gene Categories. Defined gene categories by biological process were downloaded from the Gene Ontology project (http://www.geneontology.org/); to avoid toosmall gene sets, we only considered those with at least 40 genes. 
tRNA modifications. Annotation of tRNA modifications were download from MODOMICS 5 database (http://modomics.genesilico.pl)

\section{Acknowledgements}

We are thankful to the European Research Council and the Israel Science Foundation for grant support. We thank the Pilpel and Friedman labs, and Noa Hefetz-Aharon, Martin Mikl, Schraga Schwartz, and Daniel Douek for stimulating discussions

\section{References}

1. Vibert, J. \& Thomas-Vaslin, V. Modelling T cell proliferation: Dynamics heterogeneity depending on cell differentiation, age, and genetic background. PLoS Comput. Biol. 13, e1005417 (2017).

2. Piccirillo, C. A., Bjur, E., Topisirovic, I., Sonenberg, N. \& Larsson, O. Translational control of immune responses: from transcripts to translatomes. Nat. Immunol. 15, 503-511 (2014).

3. Gingold, H. et al. A Dual Program for Translation Regulation in Cellular Proliferation and Differentiation. Cell 158, 1281-1292 (2014).

4. Rak, R., Dahan, O. \& Pilpel, Y. Repertoires of tRNAs: The Couplers of Genomics and Proteomics. Annu. Rev. Cell Dev. Biol. 34, 239-264 (2018).

5. Machnicka, M. A. et al. MODOMICS: A database of RNA modification pathways - 2013 update. Nucleic Acids Res. 41, (2013).

6. Björk, G. R. Biosynthesis and function of modified nucleosides. in tRNA: Structure, Biosynthesis, and Function 165-205 (American Society of Microbiology, 1995). doi:10.1128/9781555818333.ch11

7. Zaborske, J. M. et al. A nutrient-driven tRNA modification alters translational fidelity and genome-wide protein coding across an animal genus. PLoS Biol. 12, e1002015 (2014).

8. Novoa, E. M., Pavon-Eternod, M., Pan, T. \& Ribas de Pouplana, L. A role for tRNA modifications in genome structure and codon usage. Cell 149, 202-13 (2012).

9. Clark, W. C., Evans, M. E., Dominissini, D., Zheng, G. \& Pan, T. tRNA base methylation identification and quantification via high-throughput sequencing. RNA 22, 1771-1784 (2016).

10. Ranjan, N. \& Leidel, S. A. The epitranscriptome in translation regulation: mRNA and tRNA modifications as the two sides of the same coin? FEBS Lett. 593, 1483-1493 (2019).

11. Gamper, H. B., Masuda, I., Frenkel-Morgenstern, M. \& Hou, Y.-M. Maintenance of protein synthesis reading frame by EF-P and m1G37-tRNA. Nat. Commun. 6, 7226 (2015).

12. Tuorto, F. \& Lyko, F. Genome recoding by tRNA modifications. Open Biology 6, (2016). 
13. Waas, W. F., Druzina, Z., Hanan, M. \& Schimmel, P. Role of a tRNA base modification and its precursors in frameshifting in eukaryotes. J. Biol. Chem. 282, 26026-26034 (2007).

14. Wei, F. Y. et al. Deficit of tRNALys modification by Cdkal1 causes the development of type 2 diabetes in mice. J. Clin. Invest. 121, 3598-3608 (2011).

15. Damon, J. R., Pincus, D. \& Ploegh, H. L. tRNA thiolation links translation to stress responses in Saccharomyces cerevisiae. Mol. Biol. Cell 26, 270-282 (2015).

16. Endres, L., Dedon, P. C. \& Begley, T. J. Codon-biased translation can be regulated by wobble-base tRNA modification systems during cellular stress responses. RNA Biol. 12, 603-614 (2015).

17. Gu, C., Begley, T. J. \& Dedon, P. C. tRNA modifications regulate translation during cellular stress. FEBS Lett. 588, 4287-96 (2014).

18. Close, P., Bose, D., Chariot, A. \& Leidel, S. A. DYNAMIC REGULATION OF tRNA MODIFICATIONS IN CANCER. Cancer and Noncoding RNAs (Elsevier, 2018). doi:10.1016/B978-0-12-811022-5.00010-3

19. Rapino, F., Delaunay, S., Zhou, Z., Chariot, A. \& Close, P. tRNA Modification: Is Cancer Having a Wobble? Trends in Cancer 3, 249-252 (2017).

20. Zaborske, J. M. et al. A Nutrient-Driven tRNA Modification Alters Translational Fidelity and Genome-wide Protein Coding across an Animal Genus. PLoS Biol. 12, e1002015 (2014).

21. Biswas, P., Jiang, X., Pacchia, A. L., Dougherty, J. P. \& Peltz, S. W. The human immunodeficiency virus type 1 ribosomal frameshifting site is an invariant sequence determinant and an important target for antiviral therapy. J. Virol. 78, 2082-7 (2004).

22. Zheng, G. et al. Efficient and quantitative high-throughput tRNA sequencing. Nat. Methods 12, 835-7 (2015).

23. Lowe, T. M. \& Eddy, S. R. tRNAscan-SE: a program for improved detection of transfer RNA genes in genomic sequence. Nucleic Acids Res. 25, 955-64 (1997).

24. dos Reis, M., Savva, R. \& Wernisch, L. Solving the riddle of codon usage preferences: A test for translational selection. Nucleic Acids Res. 32, 5036-5044 (2004).

25. Novoa, E. M., Pavon-Eternod, M., Pan, T. \& Ribas De Pouplana, L. A role for tRNA modifications in genome structure and codon usage. Cell 149, 202-213 (2012).

26. de Crécy-Lagard, V. et al. Matching tRNA modifications in humans to their known and predicted enzymes. Nucleic Acids Res. 47, 2143-2159 (2019).

27. Pan, T. Modifications and functional genomics of human transfer RNA. Cell Res. 28, 395404 (2018).

28. Noma, A., Kirino, Y., Ikeuchi, Y. \& Suzuki, T. Biosynthesis of wybutosine, a hyper-modified nucleoside in eukaryotic phenylalanine tRNA. EMBO J. 25, 2142-2154 (2006).

29. Durant, P. C., Bajji, A. C., Sundaram, M., Kumar, R. K. \& Davis, D. R. Structural Effects of Hypermodified Nucleosides in the Escherichia coli and Human tRNA ${ }^{\text {Lys }}$ Anticodon 
Loop: The Effect of Nucleosides $\mathrm{s}^{2} \mathrm{U}, \mathrm{mcm}{ }^{5} \mathrm{U}, \mathrm{mcm}^{5} \mathrm{~s}^{2} \mathrm{U}, \mathrm{mnm}{ }^{5} \mathrm{~s}^{2} \mathrm{U}, \mathrm{t}^{6} \mathrm{~A}$, and $\mathrm{ms}^{2} \mathrm{t}$ ${ }^{6} \mathrm{~A}^{+}$. Biochemistry 44, 8078-8089 (2005).

30. Arthur, L. L. et al. Translational control by lysine-encoding A-rich sequences. Sci. Adv. 1, e1500154 (2015).

31. Gurvich, O. L. et al. Sequences that direct significant levels of frameshifting are frequent in coding regions of Escherichia coli. EMBO J. 22, 5941-5950 (2003).

32. Mikl, M., Alon, A., Mordret, E., Pilpel, Y. \& Segal, E. Extensive programmed ribosomal frameshifting in human as revealed by a massively parallel reporter assay. bioRxiv 469692 (2018). doi:10.1101/469692

33. Stevenson, M., Stanwick, T. L., Dempsey, M. P. \& Lamonica, C. A. HIV-1 replication is controlled at the level of T cell activation and proviral integration. EMBO J. 9, 1551-60 (1990).

34. Mordret, E. et al. Systematic Detection of Amino Acid Substitutions in Proteomes Reveals Mechanistic Basis of Ribosome Errors and Selection for Translation Fidelity. Mol. Cell 75, 427-441.e5 (2019).

35. Wohlgemuth, I., Pohl, C., Mittelstaet, J., Konevega, A. L. \& Rodnina, M. V. Evolutionary optimization of speed and accuracy of decoding on the ribosome. Philos. Trans. R. Soc. Lond. B. Biol. Sci. 366, 2979-86 (2011).

36. Kirchner, S. \& Ignatova, Z. Emerging roles of tRNA in adaptive translation, signalling dynamics and disease. Nature Reviews Genetics 16, 98-112 (2015).

37. Santos, M., Fidalgo, A., Varanda, A. S., Oliveira, C. \& Santos, M. A. S. tRNA Deregulation and Its Consequences in Cancer. Trends Mol. Med. (2019).

doi:10.1016/j.molmed.2019.05.011

38. Goodarzi, H. et al. Modulated Expression of Specific tRNAs Drives Gene Expression and Cancer Progression. Cell 165, 1416-1427 (2016).

39. Kinjyo, l. et al. Real-time tracking of cell cycle progression during CD8+ effector and memory T-cell differentiation. Nat. Commun. 6, 6301 (2015).

40. Tuorto, F. \& Lyko, F. Genome recoding by tRNA modifications. Open Biology 6, (2016).

41. Schweizer, U., Bohleber, S. \& Fradejas-Villar, N. The modified base isopentenyladenosine and its derivatives in tRNA. RNA Biol. 14, 1197-1208 (2017).

42. Salomons, F. A. et al. Selective Accumulation of Aggregation-Prone Proteasome Substrates in Response to Proteotoxic Stress. Mol. Cell. Biol. 29, 1774 LP - 1785 (2009).

43. Cotto, J. J. \& Morimoto, R. I. Stress-induced activation of the heat-shock response: cell and molecular biology of heat-shock factors. Biochem. Soc. Symp. 64, 105-118 (1999).

44. Jaitin, D. A. et al. Massively parallel single-cell RNA-seq for marker-free decomposition of tissues into cell types. Science 343, 776-9 (2014).

45. Torres, A. G., Reina, O., Stephan-Otto Attolini, C. \& Ribas de Pouplana, L. Differential expression of human tRNA genes drives the abundance of tRNA-derived fragments. Proc. 

available under aCC-BY-NC-ND 4.0 International license.

Natl. Acad. Sci. U. S. A. 116, 8451-8456 (2019).

46. Thornlow, B. P. et al. Predicting transfer RNA gene activity from sequence and genome context. Genome Res. 30, 85-94 (2020). 
Figure legends

Figure 1- Experimental design and codon usage changes during T-cell activation.

A. Schematic representation of the experimental design.

B. T-cell activation codon bias changes. Analysis of codon usage changes based on mRNA expression (normalized to amino-acid, i.e. "codon-bias") as measured by RNA-sequencing. The codons are sorted based on proliferation vs. differentiation codon usage as described in Gingold et.al ${ }^{3}$. Codons ending with $\mathrm{C}$ or $\mathrm{G}$ are marked blue, codons ending with $\mathrm{A}$ or $\mathrm{T}$ are marked red. C. Codon bias differentiates proliferative and arrested T-cells. Shown here is a principle component analysis of the samples, based on their codon bias, as presented in panel A. The first component (69\% variance explained) best separates the naïve and $20 \mathrm{~h}$ samples.

$D$ and E. Correlation of position in first PC plotted against average expression of S-phase (D), and M-phase (E) genes (Z-transformed, average of 129 and 292 genes respectively).

Figure 2- tRNA avilabilty and translation efficiency change during T-cell activation

A. Dynamics of tRNA expression during T-cell activation. Shown here is a heat-map representation of changes in tRNA expression at each time point, normalized by expression in Naïve T-cells. Rows are sorted based on the fold-change between naïve and $20 \mathrm{~h}$ after activation.

B and C- A PCA projection of the mouse codon usage of gene sets derived from GO categories. Each point represents one gene set. Gene sets corresponding to tissue-specific GO terms are to the left side, and those corresponding to proliferation related GO terms are to the right. The color code in the upper panel in each subplot displays changes at the mRNA level, averaged over all the genes in each gene set. In the middle panel in each subplot, each gene category is color coded according to the relative change in availability of the tRNAs that correspond to the codon usage of its constituent genes, averaged over all genes in the gene set. A red color for a given gene set indicates that on average the genes in that set have codons that mainly correspond to the tRNAs that are induced in the condition, whereas a blue color indicates that the codon usage in the set is biased toward the tRNAs that were repressed in that given condition. The two lower panels show, as density plots, the correlation across GO categories between expression values for each category to its tAl value. In B, the conditions are Naïve vs. T-cells $20 \mathrm{~h}$ after activation. In $\mathrm{C}$, the conditions are naïve vs. precursor memory $\mathrm{T}$-cells $72 \mathrm{~h}$ after activation.

Figure 3- Changes in tRNA modifications during T-cell activation

A. Average modification levels based on tRNA-seq from mice naïve and activated (20h) T-cells $(n=3)$. Threshold for modification calling is: minimum coverage of modified position $=20$ reads, Modification fraction above $10 \%$ in at least one sample. 
B. Two modifications: Lys-TTT at position 37 (ms2i6A, upper left panel) and Phe-GAA at position 37 (Wybutosine, lower left panel) are decreased in highly proliferating T-cells. m1A modification at position 58 remains constant throughout the activation process (right panel).

Figure 4- Reduction of wybutosine modification induces frameshift in translation.

A. A scheme for creating HeLa cells Knockout and measuring frameshift using a reporter.

B. Frameshift levels measured by GFP expression normalized to mCherry. ${ }^{*} \quad P<0.05,{ }^{* *} p<0.005$ ( $n=3$ biological repeats)

C. Changes in modification levels, measured by tRNA sequencing.

D. Changes in tRNA abundance, WT vs. tyw-1 knockout. tRNA abundance is measured by the number of reads aligned to each tRNA, grouped by anticodon.

Figure 5 - trade-off between speed and accuracy.

T-cell activation and immune-related genes are avoiding the use of UUU codons. Shown here are GO categories enriched with genes that avoid the use of UUU codon normalized to Phenylalanine usage. Analysis of other " $U$ " ending codons did not show enrichment for T-cell related GO categories (table S1 
bioRxiv preprint doi: https://doi.org/10.1101/2020.03.14.991901; this version posted March 15, 2020. The copyright holder for this preprint (which was not certified by peer review) is the author/funder, who has granted bioRxiv a license to display the preprint in perpetuity. It is made available under aCC-BY-NC-ND 4.0 International license.

A

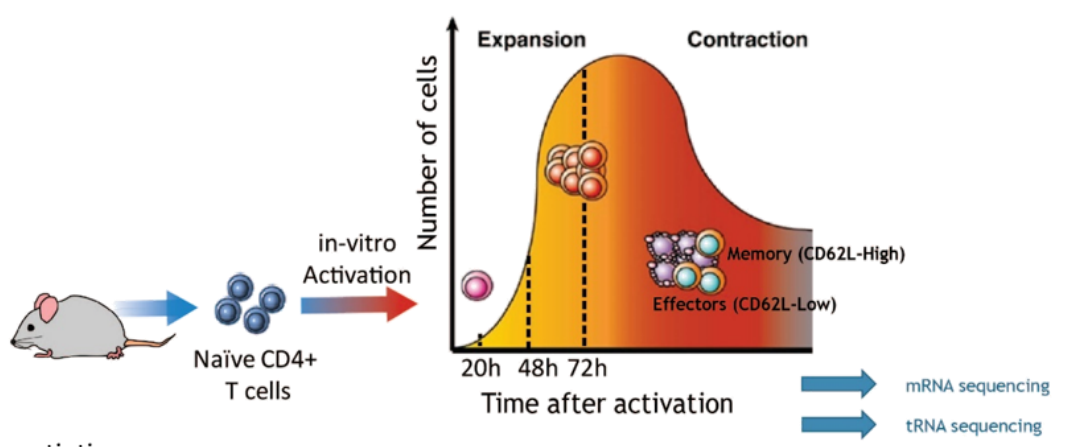

B Differentiation

codons

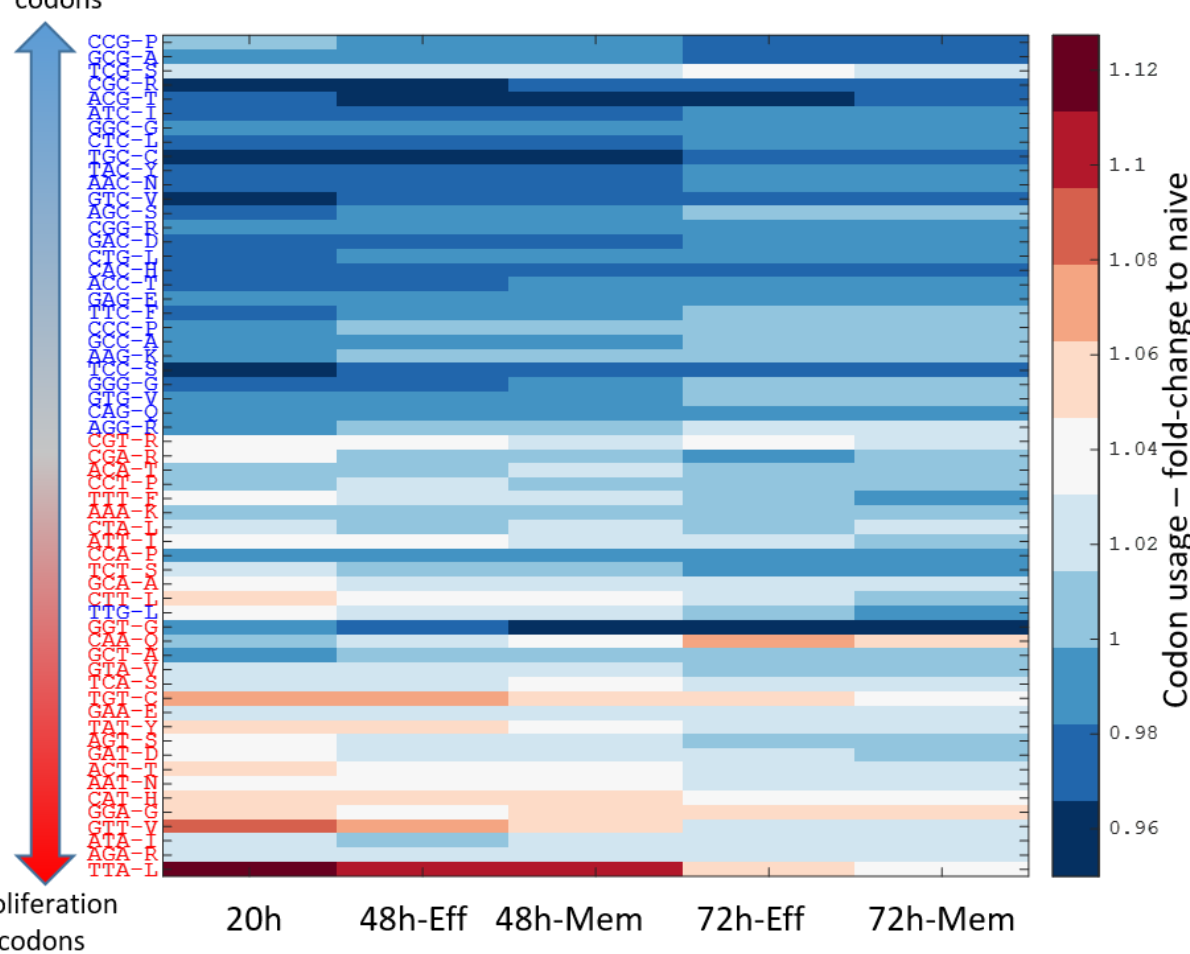

C
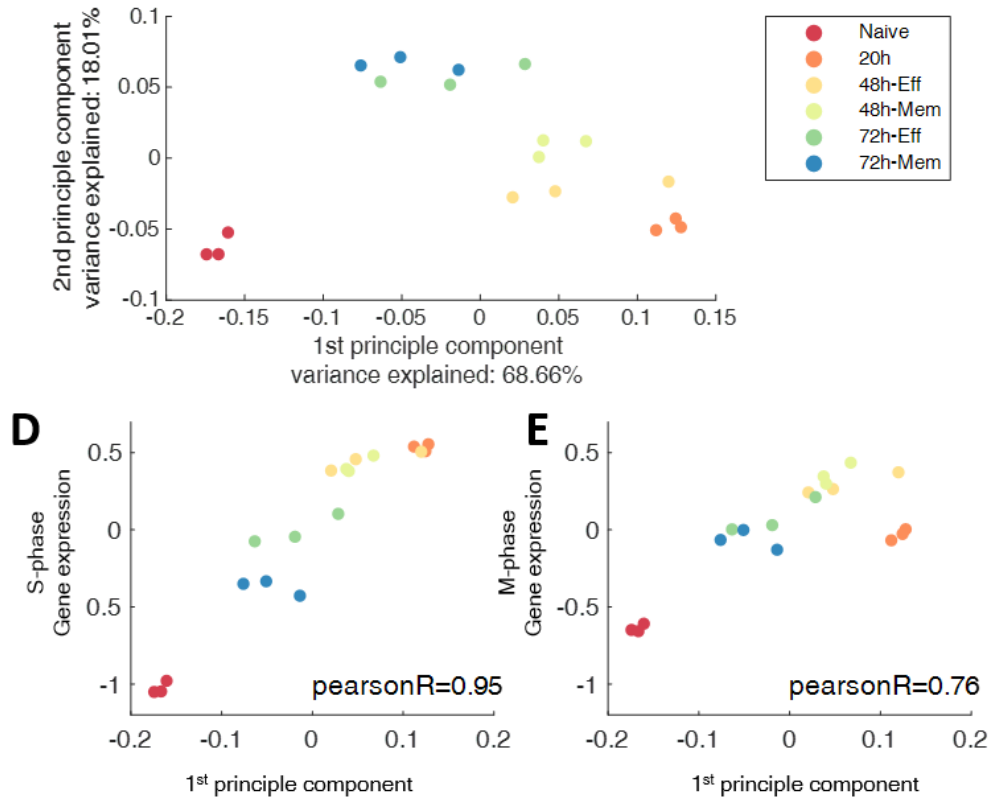
bioRxiv preprint doi: https://doi.org/10.1101/2020.03.14.991901; this version posted March 15, 2020. The copyright holder for this preprint (which was not certified by peer review) is the author/funder, who has granted bioRxiv a license to display the preprint in perpetuity. It is made available under aCC-BY-NC-ND 4.0 International license.

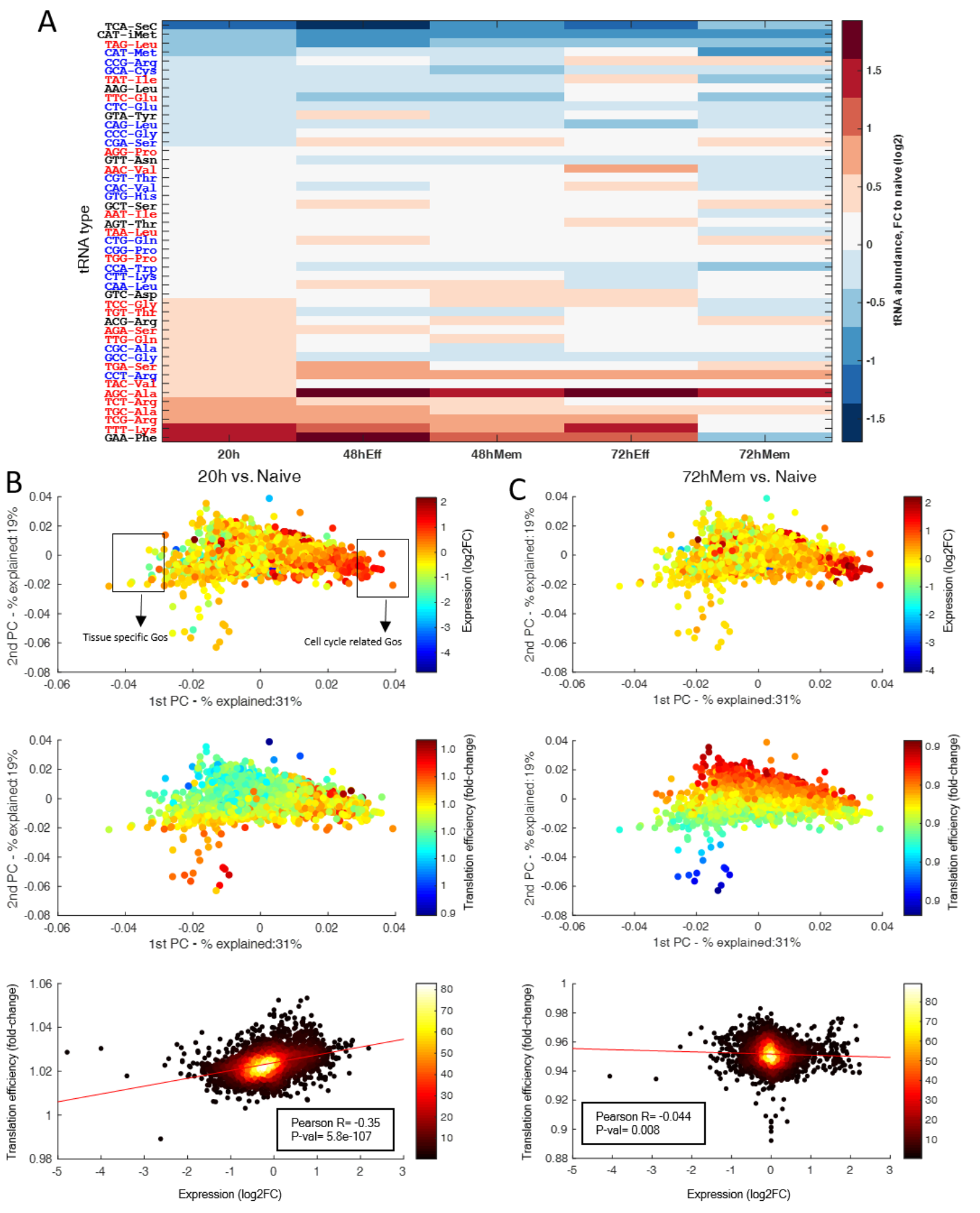




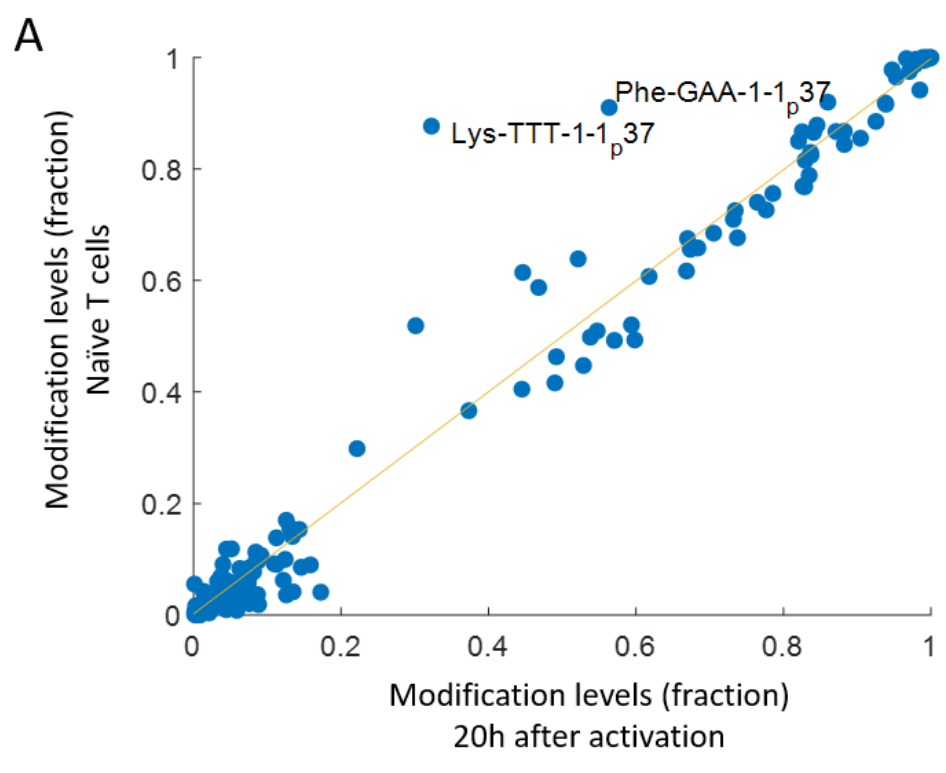

B
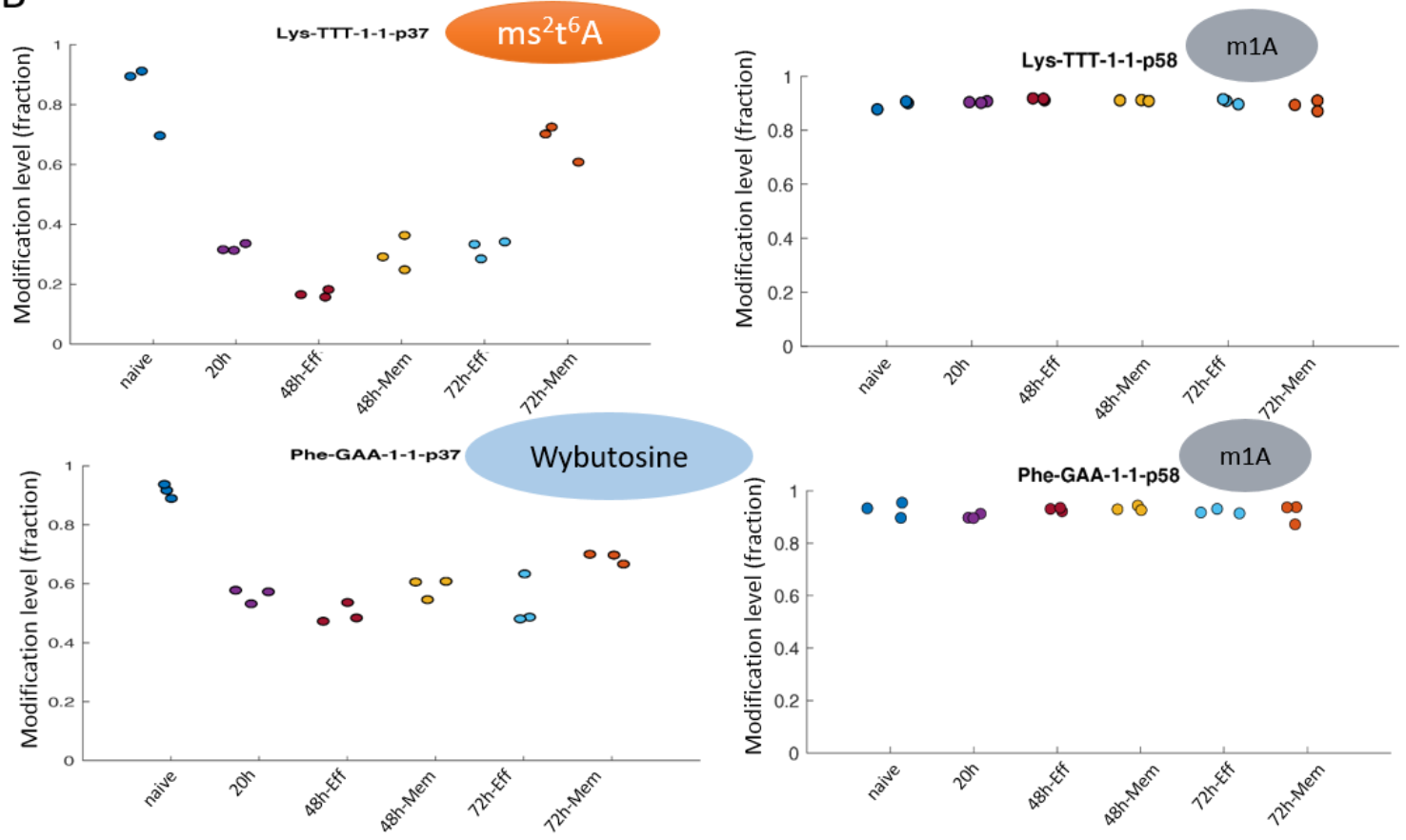


\section{A}

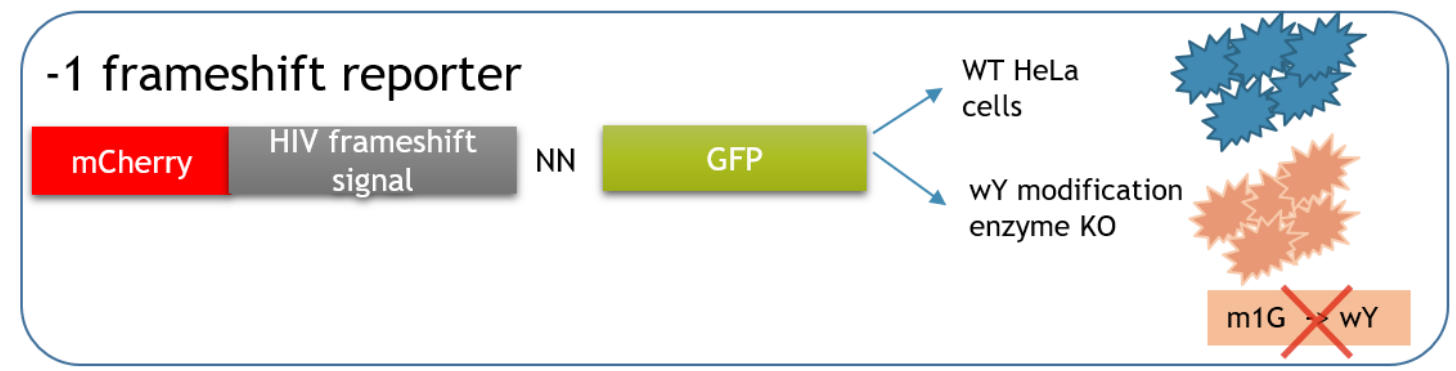

B

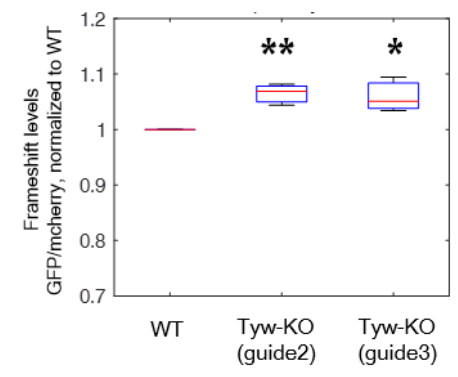

C

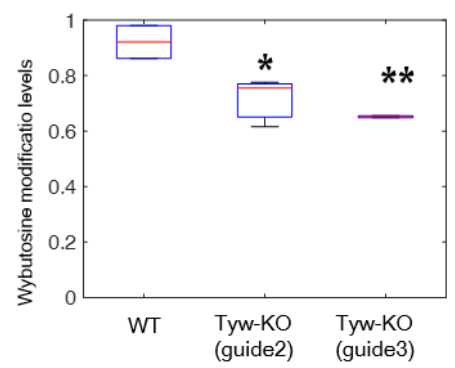

D

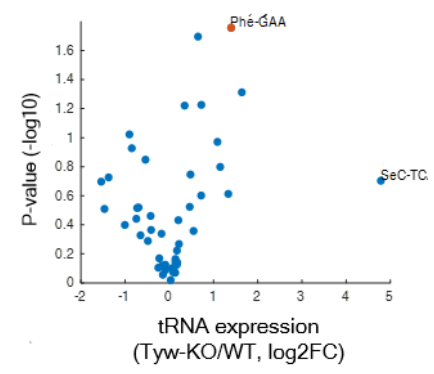


bioRxiv preprint doi: https://doi.org/10.1101/2020.03.14.991901; this version posted March 15, 2020. The copyright holder for this preprint (which was not certified by peer review) is the author/funder, who has granted bioRxiv a license to display the preprint in perpetuity. It is made available under aCC-BY-NC-ND 4.0 International license.

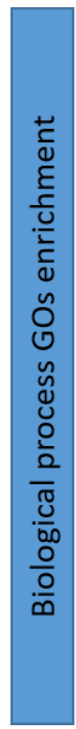

UUU avoidance

antigen processing and presentation of peptide antigen via MHC class lb canonical Wnt signaling pathway \begin{tabular}{|r|}
\hline antigen processing and presentation \\
\hline \hline positive regulation of T cell mediated immunity \\
\hline \hline antigen processing and presentation via MHC class Ib
\end{tabular} \begin{tabular}{|r|}
\hline antigen processing and presentation \\
\hline \hline positive regulation of T cell mediated immunity \\
\hline \hline antigen processing and presentation via MHC class Ib
\end{tabular}

\begin{tabular}{rr}
\hline \hline cell killing \\
\hline \hline
\end{tabular}
fat cell differentiation immune system process peroxisome membrane biogenesis killing of cells of other organism cell activation membrane biogenesis regulation of lens fiber cell differentiation ubiquitin-dependent endocytosis negative regulation of fibroblast growth factor receptor signaling pathway multi-organism catabolic process multi-organism metabolic process metabolism of substance in other organism involved in symbiotic interaction positive regulation of low-density lipoprotein receptor activity modulation of development of symbiont involved in interaction with host regulation of low-density lipoprotein particle receptor binding

nuclear retention of pre-mRNA with aberrant 3 '-ends at the site of..

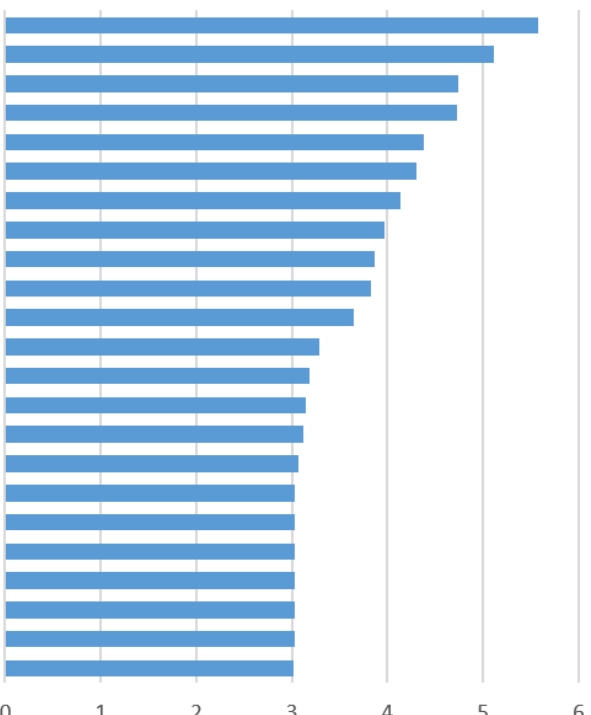

Enrichment significance (-log10 (p-val)) 


\section{SUPPORTING INFORMATION FOR}

\section{Dynamic changes in tRNA modifications and abundance during T- cell activation}

Roni Rak ${ }^{1}$, Michal Polonsky ${ }^{2}$, Inbal Eizenberg ${ }^{2}$, Orna Dahan $^{1}$, Nir Friedman ${ }^{2 *}$, Yitzhak Pilpel ${ }^{1 *}$

${ }^{1}$ Department of Molecular Genetics,

${ }^{2}$ Department of Immunology,

The Weizmann Institute of Science, Rehovot, Israel.

*corresponding authors

Corresponding Authors:

Yitzhak Pilpel, Ph.D.

Email: pilpel@weizmann.ac.il

Nir Friedman, Ph.D.

Email: nir.friedman@weizmann.ac.il

This PDF file includes:

Figures S1 to S4

Table S1 


\section{Supplementary figures}
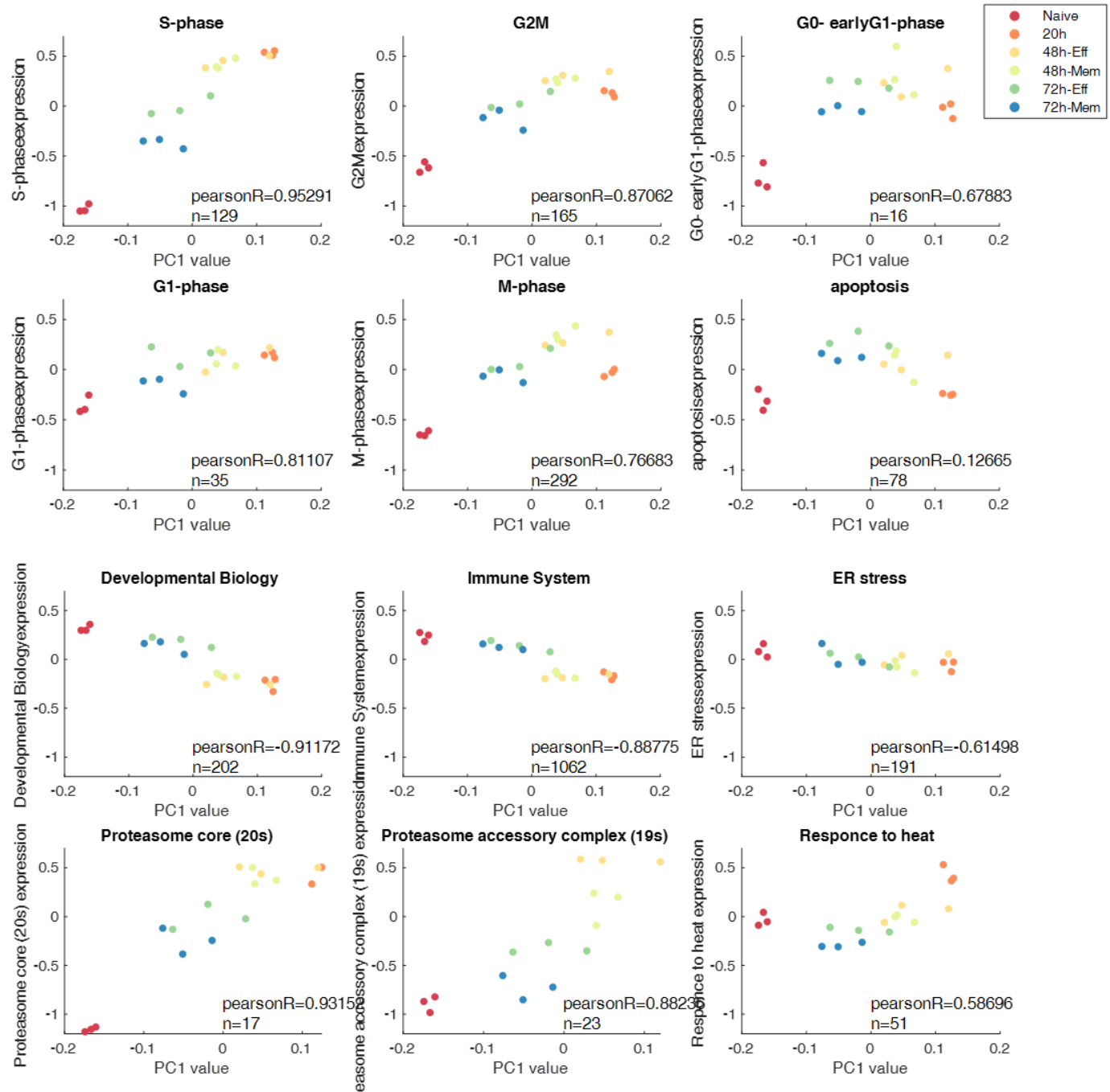

Figure 1S- Scatter plot of position in PC1 (calculated based on expressed codon usage, as in figure $1 \mathrm{C}$ ) vs average expression of the genes in the indicated GO category (Z-transformed, average of $n$ genes) 
bioRxiv preprint doi: https://doi.org/10.1101/2020.03.14.991901; this version posted March 15, 2020. The copyright holder for this preprint (which was not certified by peer review) is the author/funder, who has granted bioRxiv a license to display the preprint in perpetuity. It is made available under aCC-BY-NC-ND 4.0 International license.
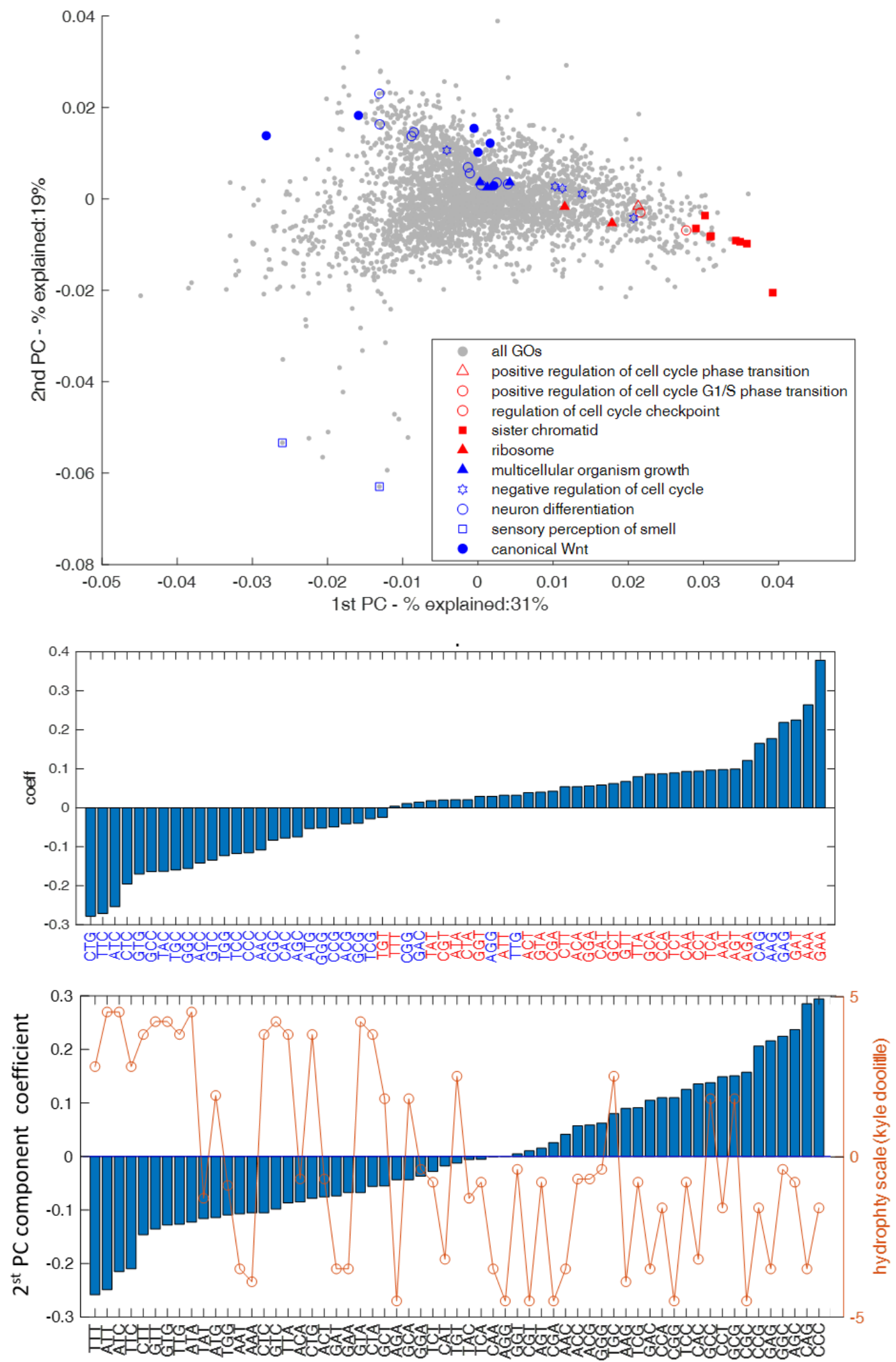

Figure 2S- A. PCA projection of the mouse codon usage of gene sets derived from GO categories. The location of each gene set in this space is determined by the average codon usage of all the genes that belong to it. The $\%$ variance, out of the total original variance in the high- 
dimensional space, spanned by the first and second PCs is indicated on the $x$ and $y$ axis, respectively. B. codon coefficiency contribute to the $1^{\text {st }} P C$. Marked in red codons end with $A / T$, marked in blue codons that end with G/C. C. codon coefficiency contribute to the $2^{\text {nd }} P C$.

A

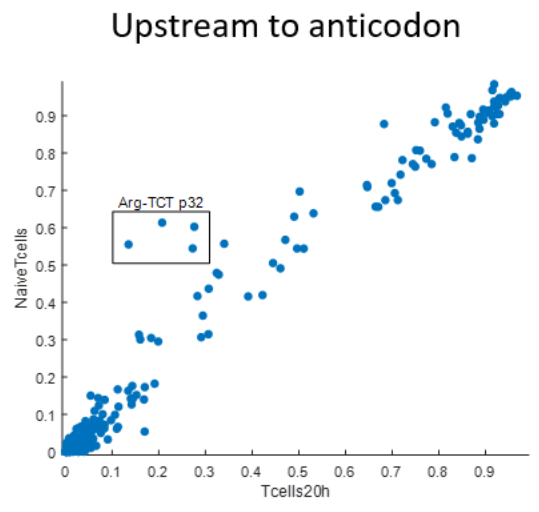

B

\section{$\mathrm{m} 1 \mathrm{G}$}

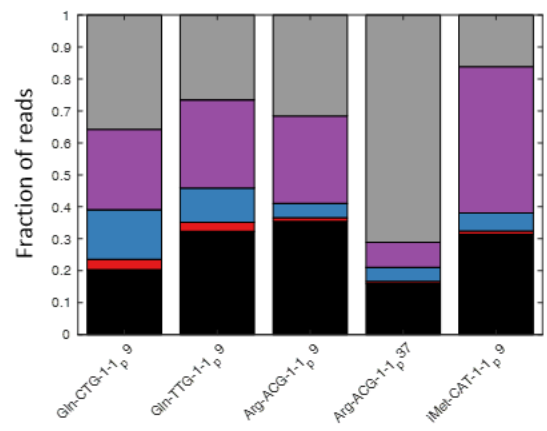

A to I

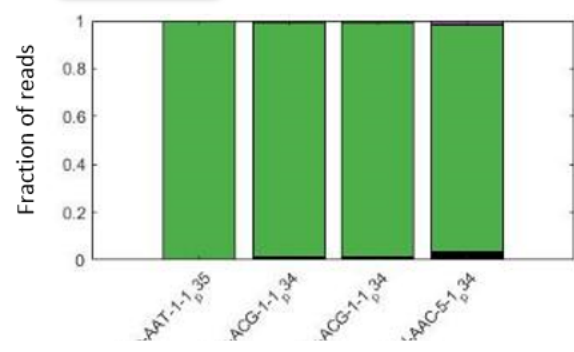

downstream to anticodon

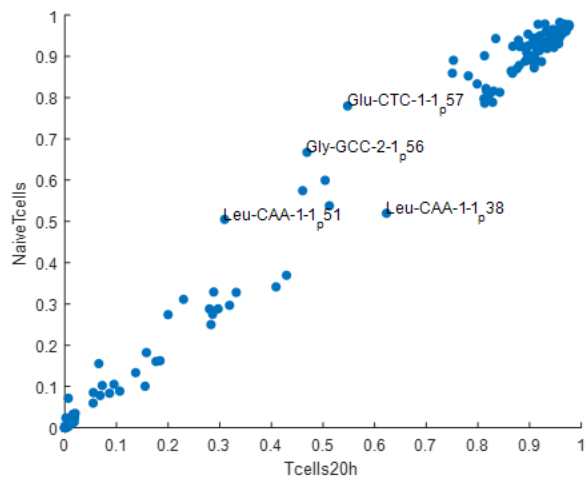

\section{$\mathrm{m} 1 \mathrm{~A}$}
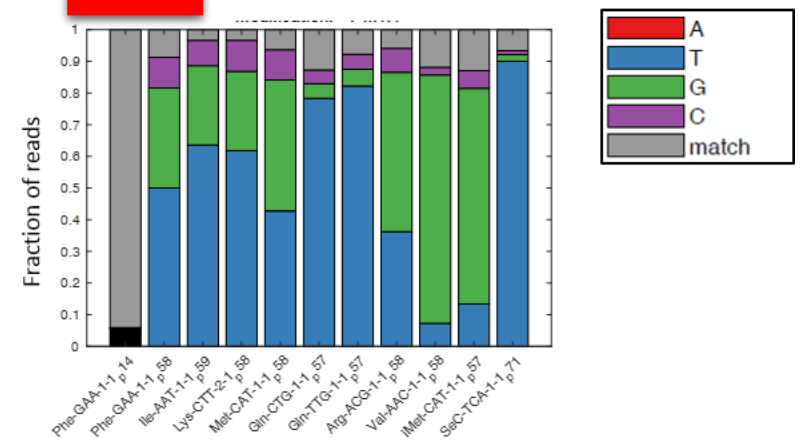

Figure 3S- modification detection based on tRNA sequencing. A. average modification levels base on tRNA-seq from mice naïve and activated (20h) T-cells $(n=3)$. Threshold for modification calling is: minimum coverage of modified position $=20$ reads, Modification fraction above $10 \%$ in at least one sample. Left panel- upstream to anticodon, right panel- downstream to anticodon. B. mismatches and RT-drops in indicated modification positions. 


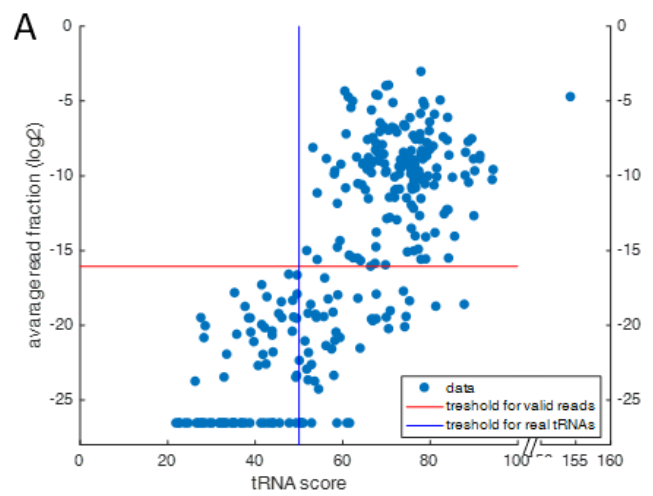

B
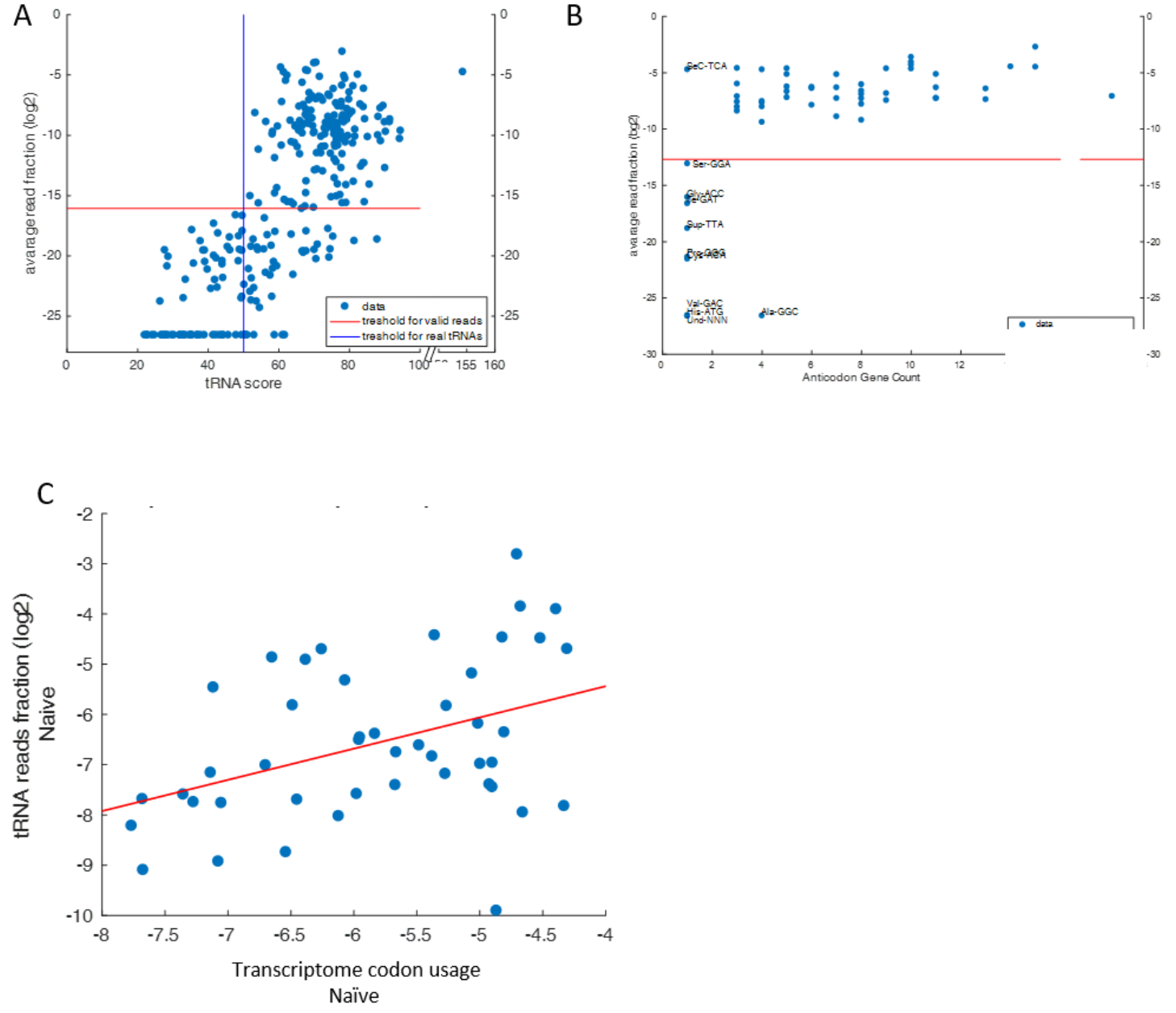

Figure 4S- tRNA Deep sequencing in T-cells shows agreement with codon usage and tRNA score

A. TRNA read count is in agreement with tRNA score. Shown here is a scatter plot of the predicted tRNA score as calculated by tRNAscan-SE compare to fraction of reads of each unique tRNA. tRNAs with score below 50 consider to be pseudo-tRNAs, and indeed gets very few reads. B. anticodon read count. Shown here is a scatter plot of the tRNA gene copy number, calculated based on the number of tRNA of the same anti-codon with score above 50, compare to fraction of reads of each tRNA anti-codon group. C. tRNA expression correlates with codon usage. Shown here is a scatter-plot indicating the correlation between tRNA read count (grouped by anti-codon) and the demand for the codon (codonome) as determined by mRNA expression in the same condition (spearman $\mathrm{R}=0.4, \mathrm{p}-\mathrm{val}=0.005$ ). 


\section{Supplementary table}

\begin{tabular}{|c|c|c|c|}
\hline $\begin{array}{l}\text { U-C box } \\
\text { (avoidance } \\
\text { of U } \\
\text { ending) }\end{array}$ & $\begin{array}{l}\text { Amino } \\
\text { Acid }\end{array}$ & Main Gos & Gorilla link \\
\hline UUU/UUC & Phe & $\begin{array}{l}\text { cell killing, antigen processing and presentation, } \\
\text { canonical Wnt signaling pathway, T cell activation }\end{array}$ & $\begin{array}{l}\text { http://cbl- } \\
\text { gorilla.cs.technion.ac.il/GOrilla/l2Ilnvn4/GOResults.html }\end{array}$ \\
\hline UAU/UAC & Tyr & $\begin{array}{l}\text { G protein-coupled receptor signaling pathway, } \\
\text { brown fat cell differentiation, regulation of } \\
\text { excretion, }\end{array}$ & $\begin{array}{l}\text { http://cbl- } \\
\text { gorilla.cs.technion.ac.il/GOrilla/108oxgsw/GOResults.html }\end{array}$ \\
\hline UGU/UGC & Cys & $\begin{array}{l}\text { regulation of renal system process, metal ion } \\
\text { homeostasis, G protein-coupled receptor } \\
\text { signaling pathway }\end{array}$ & $\begin{array}{l}\text { http://cbl- } \\
\text { gorilla.cs.technion.ac.il/GOrilla/85j8vdke/GOResults.html }\end{array}$ \\
\hline GAU/GAC & Asp & $\begin{array}{l}\text { positive regulation of small molecule metabolic } \\
\text { process, L-amino acid transport, regeneration, } \\
\text { canonical Wnt signaling pathway }\end{array}$ & $\begin{array}{l}\text { http://cbl- } \\
\text { gorilla.cs.technion.ac.il/GOrilla/2h95/roo/GOResults.html }\end{array}$ \\
\hline $\mathrm{CAU} / \mathrm{CAC}$ & $\mathrm{His}$ & $\begin{array}{l}\text { oxidative DNA demethylation, negative regulation } \\
\text { of cell fate commitment, heat generation }\end{array}$ & $\begin{array}{l}\text { http://cbl- } \\
\text { gorilla.cs.technion.ac.il/GOrilla/bq0t10hr/GOResults.html }\end{array}$ \\
\hline AGU/AGC & $\begin{array}{l}\text { Ser }(6- \\
\text { box })\end{array}$ & $\begin{array}{l}\text { regulation of carbohydrate catabolic process, } \\
\text { acrosome reaction, DNA packaging, positive } \\
\text { regulation of antigen processing and presentation }\end{array}$ & $\begin{array}{l}\text { http://cbl- } \\
\text { gorilla.cs.technion.ac.il/GOrilla/bsrxieey/GOResults.html }\end{array}$ \\
\hline AAU/AAC & Asn & $\begin{array}{l}\text { chromatin organization, fatty acid elongation, } \\
\text { negative regulation of multicellular organismal } \\
\text { process }\end{array}$ & $\begin{array}{l}\text { http://cbl- } \\
\text { gorilla.cs.technion.ac.il/GOrilla/2ggqg6k8/GOResults.html }\end{array}$ \\
\hline
\end{tabular}

Table s1- Go enrichment of genes avoiding the use of XXU vs XXC codons.

We sorted genes based on the use of the indicated codons, and preformed GO enrichment analysis using Gorilla. The significant Gos that were enriched in this analysis are presented here for each codon pair. 